\title{
Debugging of ORNL Series of Mathematical Phantoms of Human Body
}

\author{
D. KRSTIC* AND D. NikEZIC \\ Faculty of Science, University of Kragujevac, R. Domanovic 12, 34000 Kragujevac, Serbia
}

(Received March 24, 2010; in final form October 22, 2010)

\begin{abstract}
Series of mathematical phantoms of human body, given by Oak Ridge National Laboratory (ORNL), was programmed as input files for MCNP-4B code. Detailed check of geometry of these phantoms performed by MCNP-4B, discovered some minor errors, that resulted in overlapping of some organs. Three types of errors were found and described here: (a) colon overlaps with pelvis bone; (b) facial skeleton penetrate the head skin, and (c) esophagus overlaps with stomach. These errors prevent correct execution of program. Proposal for correction of these errors are given in this paper.
\end{abstract}

PACS: 02.50.Ng, 07.05.Tp, 87.53.Bn

\section{Introduction}

Computational models of human body (the series of ORNL phantoms) have been used extensively with radiation transport codes to estimate organ doses resulting from diagnostic radiology examinations, for the planning of radiotherapy treatment, for the calculation of radiation protection quantities and environmental radiation exposures [1-4]. Frequently used phantoms are: MIRD-5 [5] and sex-specific adult phantoms of MIRD type, ADAM and EVA [6].

Recently a new generation of body models used computed tomography (CT) or magnetic resonance (MR) data of real persons $[7-10]$.

The ORNL phantoms have been used, and a few modifications are made by [11, 12]. In Ref. [12] extensive changes have been made to the ORNL phantoms to make them more compliant with ICRP Publication 89 [13]. Input files with these phantoms for MCNP code were given at the MCNP medical physics library [14].

Mathematical phantoms of human body were given on Oak Ridge National Laboratory (ORNL) web site [15]. There, a series of phantoms was presented, including adult male, adult female, and individuals of age 1, 5, 10 and 15 years old as well as newborn.

Input file for MCNP-4B (Monte Carlo N-Particle) [16] with ORNL of human phantoms of adult male and female were prepared earlier by authors, with the intention to calculate an absorbed dose from ${ }^{137} \mathrm{Cs}$ in soil [17]. Later, the input files were prepared for ORNL series of phantoms (newborn, 1 year, 5 years, 10 years, 15 years models) [18]. Recommendations given by ORNL regarding the dimensions, shape, chemical constitution etc., were strictly respected in preparation of input files.

MCNP-4B code performs rigid check of geometry. During this check the problematical surfaces could be

* corresponding author; e-mail: dragana@kg.ac.rs seen in graphical output of MCNP-4B as red dashed lines. Input file with adult mathematical phantom, prepared in this work, passed MCNP-4B test and calculations with different sources and various irradiation conditions become possible. However, when MCNP-4B checked input files with other phantoms some problems in geometry appeared. The execution of MCNP-4B was not possible with error in geometry or it is constrained by the number of lost particles.

Similar, commercially available software, called "BodyBuilder" was based on report by Cristy and Eckerman [19]. It was developed to calculate the doses absorbed in specific organs from the radioactive source in some other organ. Some problems in geometry was also reported on http://www. whiterockscience.com/ bodybuilder/errata.html. Problems are related with: overlapping of organs in 5 years old model (small intestine, ascending colon, and transverse colon); in 9 months old pregnant model; 15 year female model (an extremely small overlap was found in the transverse colon); newborn transverse colon volume; liver cell description; overlapping of the kidneys and adrenals in the newborn model; overlapping between the legs and some difficulties in height and weight of the model after inclusion the neck.

Here we reported some other problems that we found during the development of our own input files for MCNP with ORNL series of human phantoms.

\section{Errors in ORNL mathematical phantom of human body}

To facilitate the understanding of the revealed "errors" the description of coordinate system is shortly given. Origin is taken at the centre of the base of trunk section, $z$ axis is directed vertically upward; $x$ axis is directed to the phantoms left, and $y$ axis is directed to the back of the phantom. All dimensions are given in centimetres. 
Three groups of errors were discovered by MCNP-4B in all phantoms (except adult male where no error was found); the errors are related to (a) colon, (b) facial skeleton and (c) stomach.

\subsection{Colon}

Segment of input file, written in this work and in recent publications [17, 18], which represents colon with original ORNL data, for the age of 15 years, is given as $52 \mathrm{pz} 21.63 \quad$ Send ascending colon

61 ty $2.590 \% .865 .161 .181 .76 \quad$ \$sigmoid colon - portion of upper torus

62 ty 2.5907 .865 .160 .591 .17

Here, 52, 61 and 62 are number of surfaces in input file (this was defined by the authors who prepared input file), $p z$ is horizontal plane with equation $z=21.63$ defining the colon upper end, ty are tori with the center at $x_{0}=2.59, y_{0}=0$ and $z_{0}=7.86$. Large radius of tori is $R=5.16$. Next two digits, 1.18 and 1.76 are axis of ellipse (two elliptical tori represent colon). Second torus (surface 62) is internal surface of colon. Actually colon is defined between tori given by surfaces 61 and 62 .

MCNP-4B enables graphical output which is cross-section between the geometry defined in input file and a plane chosen by user. The previous segment of input file and cross with the plane $y=0.68$ is presented in Fig. 1 . Here is seen that the part of colon overlaps with pelvis bone (thick vertical line).
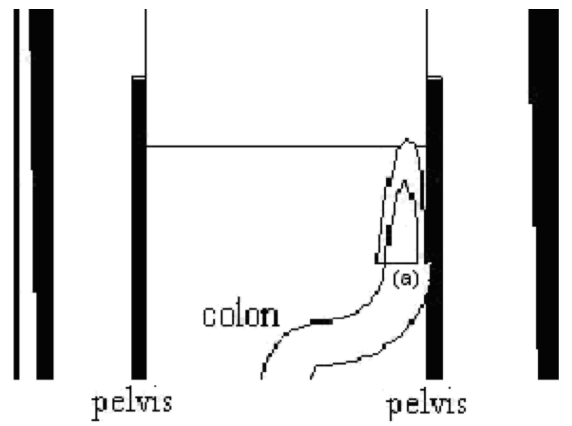

Fig. 1. Overlapping of the colon and pelvis bone in phantoms of 15 years age. Obtained by using original ORNL data. The graphical output from MCNP-4B software. Only "(a)" was inserted by additional editing and represents the first error (a). Cross with the plane $y=0.68$.

Similar problem arose in all other phantoms (excluding phantom of adult male); the extension of overlapping is not the same in different phantoms. Since the figures obtained for other phantoms are rather similar they are not given here. This problem could be resolved with minor changing of the tori center, which will shift colon for some small amount in order to avoid overlapping. In ORNL recommendation [15], table is given with data defining tori for colon. A part of the table is presented here in Table I.
TABLE I

Part of original ORNL table for colon, and values proposed here. Corrections are given in bold and italic.

\begin{tabular}{l|c|c}
\hline \hline \multicolumn{1}{c|}{ Phantom } & $x_{0}(\mathrm{ORNL})$ & $x_{0}$ (this paper) \\
\hline newborn & 0.95 & $\boldsymbol{0 . 7 5}$ \\
age 1 & 1.32 & $\mathbf{0 . 9 5}$ \\
age 5 & 1.72 & $\mathbf{1 . 3 2}$ \\
age 10 & 2.09 & $\mathbf{1 . 7 2}$ \\
age 15 (adult female) & 2.59 & $\mathbf{2 . 0 9}$ \\
adult male & \multicolumn{2}{|c}{3.00 (no need correction) }
\end{tabular}

MCNP-4B graphical output of 15 year old phantom with correction presented in Table I is given in Fig. 2. One can see that the colon is shifted without overlapping with pelvis bone.

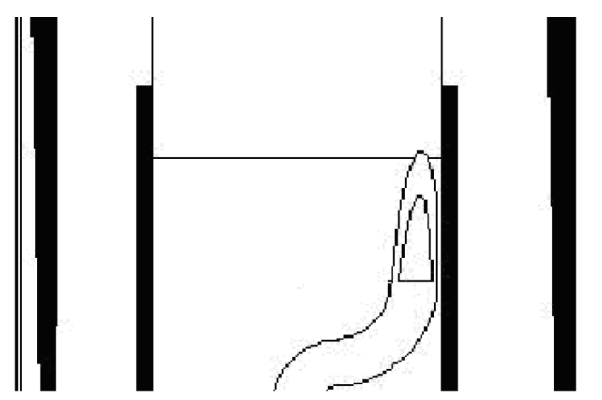

Fig. 2. Colon corrected according to Table I. There is no overlapping between colon and pelvis bone. Graphical output from MCNP-4B code.

\subsection{Facial skeleton}

The problems in facial skeleton appear in phantoms of age 10 years, 5 years, 1 year and newborn. Some facial bones penetrate the head skin. MCNP-4B shows the error in this place and disables the calculation after the number of lost particles exceeds 10 .

Facial skeleton is represented in ORNL phantom by a portion of the volume between two concentric elliptical cylinders. The cylinder is limited on the upper end by a plane. This plane is shifted up for a little and this causes the penetration of bone into the head skin. Data on facial skeleton were given in original ORNL document [15]. The

TABLE II

Part of original ORNL table for facial skeleton, and values proposed here. Corrections are given in bold and italic.

\begin{tabular}{l|c|c}
\hline \hline \multicolumn{1}{c|}{ Phantom } & $x_{0}(\mathrm{ORNL})$ & $x_{0}$ (this paper) \\
\hline newborn & 8.18 & $\mathbf{8 . 0 0}$ \\
age 1 & 11.18 & $\mathbf{1 1 . 0 8}$ \\
age 5 & 12.57 & $\mathbf{1 2 . 0 7}$ \\
age 10 & 13.73 & $\mathbf{1 3 . 5 3}$ \\
age 15 (adult female) & 14.05 (no need correction) \\
adult male & \multicolumn{2}{|c}{14.73 (no need correction) }
\end{tabular}


part of their table is given in Table II of this work. The limiting plane was designated by $z_{5}$.

\subsection{Stomach}

The error appears in phantoms of newborn, 1 and 10 years age. The error is caused by overlapping of esophagus and stomach. This problem can be resolved by small shifting of two ellipsoids which represent the stomach. Part of original table published by ORNL [15], as well as our proposal are given in Table III.

\section{TABLE III}

Part of original ORNL table for stomach, and values proposed here. Corrections are given in bold and italic.

\begin{tabular}{l|c|c|c|c|c|c}
\hline \hline \multirow{2}{*}{ Phantom } & \multicolumn{3}{|c|}{ Original ORNL } & \multicolumn{3}{c}{ Proposed here } \\
\cline { 2 - 7 } & $x_{0}$ & $y_{0}$ & $z_{0}$ & $x_{0}$ & $y_{0}$ & $z_{0}$ \\
\hline newborn & 2.54 & -1.96 & 10.80 & $\mathbf{3 . 3 6}$ & $\mathbf{- 1 . 6 6}$ & $\mathbf{1 0 . 7 0}$ \\
age 1 & 3.52 & -2.70 & 15.36 & $\mathbf{3 . 5 3}$ & -2.70 & 15.36 \\
age 10 & 5.56 & -3.51 & 25.40 & $\mathbf{5 . 9 6}$ & -3.51 & 25.40
\end{tabular}

\section{Conclusion}

Detailed study of ORNL phantom and rigid check performed by MCNP-4B code discovered some minor errors in geometry of some age dependent phantoms. The dose calculated with original ORNL phantoms and with phantoms + correction introduced here would not be very different. However, when MCNP-4B was executed with the errors in geometry described above, some particles would be "lost" in problematical area of geometry. When the number of lost particles exceeds 10, the program will terminate the further execution of simulation. Corrections of observed errors were proposed in this work. Input files with corrected geometry could be used for all versions of MCNP as well as MCNPX codes.

\section{Acknowledgments}

The authors would like to thank the Serbian Ministry of Science Technology and Development, which supported this work through project No. 171021 .

\section{References}

[1] K.F. Eckerman, J.C. Ryman, External Exposure to Radionuclides in Air, Water and Soil, US. Environmental Protection Agency, Federal Guidance Report No 12, Washington (DC) 1993.

[2] W. Bolch, B. Pomije, J. Sessions, M. Arreola, J. Williams, Med. Phys. 30, 667 (2003).

[3] R. Staton, F. Pazik, J. Nipper, J. Williams, W. Bolch, Phys. Med. Biol. 48, 805 (2003).

[4] G. Gialousis, E.N. Yakoumakis, D. I. Papadopoulou, T.K. Makri, N.E. Yakoumakis, P.A. Dimitriou, E.K. Georgiou, Phys. Med. Biol. 51, 287 (2006).

[5] W.S. Snyder, M.R. Ford, G.G. Warner, H.L. Fisher, MIRD Pamphlet No. 5, Revised, The Society of Nuclear Medicine, New York 1978.
[6] R. Kramer, M. Zankl, G. Williams, G. Drexler, GS $F$-Report S-885, GSF-National Research Center for Environment and Health, Neuherberg 1982.

[7] N. Petoussi-Henss, M. Zankl, U. Fill, D. Regulla, Phys. Med. Biol. 47, 89 (2002).

[8] M. Caon, Rad. Environ. Biophys. 42, 229 (2004).

[9] P. Ferrar, G. Gualdrini, Phys. Med. Biol. 50, 4299 (2005).

[10] R. Kramer, H.J. Khoury, J.W. Vieira, Phys. Med. Biol. 50, 5105 (2005).

[11] D.P. Cho, Radiat. Prot. Dosim. 104, 5 (2003).

[12] E.Y. Han, W.E. Bolch, K.F. Eckerman, Health Phys. 90, 337 (2006).

[13] ICRP89 International Commission on Radiological Protection, Basic anatomical and physiological data for use in radiological protection: reference values, Annals of ICRP23, Pergamon Press, Oxford 2002.

[14] T. Goorley, MCNP Medical Physics Geometry Database, Los Alamos National Laboratory, 2008. http://mcnp-green.lanl.gov/publication/ mcnp_publications.html\#medicalphysics .

[15] K.F. Eckerman, M. Cristy, J.C. Ryman, The ORNL mathematical phantom series, available at: http://homer.ornl.gov/vlab/mird2.pdf , 1996.

[16] J.F. Briesmeister, MCNP - A General Monte Carlo N-Particle Transport Code, version 4B. Los Alamos National Laboratory Report LA-12625-M, Los Alamos, New Mexico 1997.

[17] D. Krstic, D. Nikezic, Health Phys. 91, 249 (2006).

[18] D. Krstic, D. Nikezic, Comp. Phys. Commun. 17, 33 (2007).

[19] M. Cristy, K.F. Eckerman, Oak Ridge National Laboratory, Report ORNL/TM-8381/VI, 1987. 